INPLASY

PROTOCOL

To cite: Zhang et al. A Metaanalysis of the Effect of Hip Muscle Enhancement on Knee Osteoarthritis. Inplasy protocol 202150001. doi:

10.37766/inplasy2021.5.0001

Received: 30 April 2021

Published: 01 May 2021

Corresponding author:

Zhang Qiuyan

1483506234@qq.com

Author Affiliation:

Hunan University of

Technology

Support: Open Research

Foundation of Hunan

Provincial Key Laboratory,

No.2020TP1024.

Review Stage at time of this submission: Data analysis.

Conflicts of interest:

None declared.

\section{A Meta-analysis of the Effect of Hip Muscle Enhancement on Knee Osteoarthritis}

Zhang, Q1; Gao, N2; Wang, G3; Wang, Y4.

Review question / Objective: 1 - Study type: Randomized controlled trial (RCT); 2 - Subjects: patients diagnosed with knee osteoarthritis according to the American College of Rheumatology standards; 3 - Intervention measures: hip joint muscle strengthening and quadriceps femoris training control or hip joint muscle strengthening auxiliary means and auxiliary means control; 4 - Evaluation indexes: Visual Analogue Scale (VAS), Western Ontario and McMaster University Osteoarthritis Index (WOMAC) score, 6-minute walk test (6MWT), peak torque (PT), knee injury and osteoarthritis outcome score (KOOS).

Condition being studied: The Effect of Hip Muscle Enhancement on Knee Osteoarthritis. To systematically evaluate the therapeutic effect of hip joint muscle strengthening on knee osteoarthritis. Methods: CNKI, VIP, Wanfang, CBM, PubMed, The Cochrane Library, Embase and other databases were searched, and randomized controlled trials (RCTs) of hip muscle enhancement for improvement of knee osteoarthritis symptoms were collected. The included literatures were screened, data extracted, quality assessment and risk assessment, and Meta-analysis was performed using RevMan 5.3 software.

INPLASY registration number: This protocol was registered with the International Platform of Registered Systematic Review and Meta-Analysis Protocols (INPLASY) on 30 April 2021 and was last updated on 01 May 2021 (registration number INPLASY202150001).

\section{INTRODUCTION}

Review question / Objective: 1 - Study type: Randomized controlled trial (RCT); 2 Subjects: patients diagnosed with knee osteoarthritis according to the American College of Rheumatology standards; 3 -
Intervention measures: hip joint muscle strengthening and quadriceps femoris training control or hip joint muscle strengthening auxiliary means and auxiliary means control; 4 - Evaluation indexes: Visual Analogue Scale (VAS), Western Ontario and McMaster University 
Osteoarthritis Index (WOMAC) score, 6minute walk test (6MWT), peak torque (PT), knee injury and osteoarthritis outcome score (KOOS).

Condition being studied: The Effect of Hip Muscle Enhancement on Knee Osteoarthritis. To systematically evaluate the therapeutic effect of hip joint muscle strengthening on knee osteoarthritis. Methods: CNKI, VIP, Wanfang, CBM, PubMed, The Cochrane Library, Embase and other databases were searched, and randomized controlled trials (RCTs) of hip muscle enhancement for improvement of knee osteoarthritis symptoms were collected. The included literatures were screened, data extracted, quality assessment and risk assessment, and Meta-analysis was performed using RevMan 5.3 software.

\section{METHODS}

Participant or population: According to the standard of American College of Rheumatology, the patient was diagnosed with knee osteoarthritis.

Intervention: Combined hip muscle enhancement/hip muscle enhancement adjuvant.

Comparator: Blanks/quadriceps exercises/ other AIDS.

Study designs to be included: Randomized Controlled Trial (RCT).

Eligibility criteria: 1 - Study type: Randomized controlled trial; 2 - Subjects: patients diagnosed with knee osteoarthritis according to the American College of Rheumatology standards; 3 - Intervention measures: hip joint muscle strengthening and quadriceps femoris training control or hip joint muscle strengthening auxiliary means and auxiliary means control; 4 Evaluation indexes: Visual Analogue Scale (VAS), Western Ontario and McMaster University Osteoarthritis Index (WOMAC) score, 6-minute walk test (6MWT), peak torque(PT), knee injury and osteoarthritis outcome score (KOOS).
Information sources: Electronic databases, links to authors, trial registrations.

Main outcome(s): Evaluation indicators: Visual Analogue Scale, VAS, Western Ontario and McMaster University Osteoarthritis Index (WOMAC) score (including physical impairment, pain, and stiffness), 6MWT, Peak Torque(PT), knee injury and osteoarthritis outcome score (KOOS).

Quality assessment / Risk of bias analysis: Cochrane bias risk assessment and PEDro scale were used to evaluate the literature quality.

Strategy of data synthesis: In this study, only continuous variables were involved, and weighted mean difference (MD) was used to combine statistics. For variables with large mean differences and different units among studies, standard mean difference (SMD) was used to eliminate the influence of large mean differences and different units among studies on results. The $95 \%$ confidence interval $(\mathrm{Cl})$ was used for the test statistics. When $P<0.05$, there were differences in all indicators, which was of significant significance.

Subgroup analysis: According to different intervention measures; 2, according to the different types of knee osteoarthritis; 3 . Subgroup analysis was conducted according to the course of disease.

Sensitivity analysis: Sensitivity analysis was conducted by removing references one by one.

Country(ies) involved: China, the United States, the United Kingdom.

Keywords: Hip muscle enhancement; Knee osteoarthritis; Meta analysis.

Contributions of each author:

Author 1 - Zhang Qiuyan.

Author 2 - Gao Ning.

Author 3 - Wang Guojun.

Author 4 - Wang Yongjun. 